Garre-Olmo J, Vilalta-Franch J, Calvó-Perxas L, Turró-Garriga O, Conde-Sala JL, López-Pousa S, on behalf of the CoDep-AD Study Group A path analysis of patient dependence and caregiver burden in Alzheimer's disease. International Psychogeriatrics 2016; 28(7): 1133-1141.

doi:10.1017/S1041610216000223

\title{
A path analysis of dependence and caregiver burden in Alzheimer's
}

\section{disease}

Garre-Olmo, $\mathrm{J}^{1}$, Vilalta-Franch $\mathrm{J}^{1-2}$, Calvó-Perxas $\mathrm{L}^{1}$, Turró-Garriga $\mathrm{O}^{2}$, Conde-Sala $\mathrm{L}^{3}$, López-Pousa $\mathrm{S}^{1-2}$, on behalf of the CoDep-AD Study Group*

Affiliations: ${ }^{1}$ Research Unit. Institut d'Assistència Sanitària. Girona; ${ }^{2}$ Dementia Unit, Hospital de Santa Caterina, Salt; 3

Corresponding author: Josep Garre-Olmo, PhD; C/ Dr Castany s/n; 17190 Salt (Girona) Spain; Tel.: \#34-972-182600 (extension 1833); Fax: \#34-972-189017; E-mail: josep.garre@ias.scs.es 


\section{* The Cost of Dependence Associated to Alzheimer's disease in Spain Study Group (the CoDep-AD Study Group) consists of the following investigators:}

Miquel Aguilar. Hospital Mútua de Terrassa. Terrassa (Catalunya)

Jordi Alom. Hospital General Universitario de Elche. Elche (Comunitat Valenciana)

Guillermo Amer. Hospital Univeritari Son Espases. Palma de Mallorca (Balears)

Carmen Antúnez. Hospital Virgen de la Arrixaca. El Palmar (Murcia)

Rafael Arroyo. Clínica Quirón. Barcelona (Catalunya)

Miguel Barquero. Hospital La Fe. Valencia (Comunitat Valenciana)

Félix Bermejo. Hospital 12 de Octubre. Madrid (Madrid)

Fernando Castellanos. Hospital Virgen del Puerto. Plasencia (Cáceres)

Manuel Franco. Hospital de Zamora. Zamora (Castilla y León)

Guillermo García. Hospital Ramón y Cajal. Madrid (Madrid)

José María García. Instituto Andaluz de Neurociencia y Conducta. Málaga (Andalucía)

Domènec Gil. Hospital Universitari Sagrat Cor de Jesús. Barcelo9na (Catalunya)

Pedro Gil. Hospital Clínico San Carlos. Madrid (Madrid)

Albert Lleó. Hospital de Sant Pau. Barcelona (Catalunya)

Manuel Martín. Clinica Psiquiátrica Padre Menni. Pamplona (Navarraa)

Dolores Martínez. Hospital La Magdalena. Catelló de la Plana (Comunitat Valenciana)

Raimundo Mateos. Hospital de Santiago de Compostela. Santiago (Galicia)

Vicente Medrano. Hospital General Universitario de Elda. Elda (Comunitat Valenciana)

Jordi Peña. Hospital del Mar. Barcelona (Catalunya)

Pilar Quílez. Hospital Mútua de Terrassa. Terrassa (Catalunya) 


\section{ABSTRACT}

Background: Caregivers for patients with Alzheimer's disease (AD) experience high levels or burden. Our objective was to identify the primary and contextual stressors that contribute to the caregiver burden according to the conceptual framework of dependence. Methods: Observational and cross-sectional multicenter study. The sample consisted of patients with $\mathrm{AD}$ recruited in outpatient consultation offices by a consecutive sampling procedure stratified by dementia severity. Cognitive and functional status, behavior, dependence, medical comorbidities, and caregiver burden were assessed by using standardized instruments. A path analysis was approach was used to model the hypothesized direct and indirect relationships among stressors, dependence and caregiver burden according to the theoretically-based models of dependence and caregiver burden. Results: The sample consisted of 306 patients (33.3\% mild, $35.9 \%$ moderate, $30.7 \%$ severe), the mean age was 78.5 years ( $\mathrm{SD}=7.8$ ), and $66.2 \%$ were women. The model that fitted properly with the data, explaining 32\% of the caregiver burden variance, included the level of dependence, the disease severity, and the distress related to behavioral disturbances as primary stressors. The caregiver's age, gender, job status, and living together with the patient were the contextual factors related to caregiver burden. Functional disability was indirectly associated with caregiver burden via dependence and disease severity, and frequency of behavioral disturbances was indirectly associated via distress Conclusion: Dependence was, apart from behavioral disturbances, the most important primary stressor directly related to caregiver burden irrespective of the disease severity.

Keywords: Alzheimer’s disease; Dementia; Dependence; Caregiver burden 


\section{INTRODUCTION}

Alzheimer's disease (AD), the most common cause of dementia, is characterized by a progressive cognitive and functional impairment, as well as by behavioral and psychological symptoms of dementia (BPSD) that appear and fluctuate, with remissions and recurrences, through the course of the disease [1-2]. As the disease progresses, and the level of dependence increases, patients need higher levels of assistance with the activities of daily living. Usually, patients are cared for at own home, primarily by their partners and adult children, that adopt the role of primary caregivers since first stages, when memory deficits are mild, until the late-stages, when patients are absolutely dependents [3]. The process of caregiving can take several years, and has physical, psychological, social and economic consequences for the individuals that adopt the role of caregiving [4]. In this sense, there is a large body of literature that has showed that the caregiving process is associated with multiple negative outcomes. For example, caregivers use a higher number of health services compared to non-caregivers [5-6], are at higher risk to develop a depressive or anxious disorder [7], have an increased consumption of psychotropic medications [8], have poor sleep quality [9], have less time for leisure and family [10], and are at greatest risk of social isolation [11].

The caregiver burden is a multidimensional construct, which has been defined as a subjective measure of the physical, psychosocial, and economic strain experienced by individuals that take care of patients with AD [12]. The Zarit Burden Interview (ZBI) [13] is a 22-item questionnaire which has appropriate psychometric characteristics, and is the most widely used to measure burden in AD caregivers [14]. According to the specific model for dementia caregiver burden the context, the stressors, the moderators, 
and the appraisal of the caregiver should be considered [15-16]. The contextual factors include the caregiver's socioeconomic status and demographics, the ethnicity-culture, the health system, and the geography. The stressors are divided into primary stressors, that include the patient's clinical characteristics, and care situation; and secondary stressors, that include factors attributable directly to caring, and include interference with work, financial strain, and family conflict, among others. The moderators, that can exacerbate or ameliorate the burden, include the psychosocial moderators, such as personality traits, caregiver's physical health, perceived self-efficacy, information about dementia, and coping strategies, and contextual moderators such as formal service use, and availability of informal assistance. The appraisal corresponds to the caregiver's subjective assessment of their role, and according to this theoretical model, the differences among individuals in similar situations are due to the dynamic interaction between the specific caregiver resources, their vulnerabilities, and the patient care needs and demands [15-16].

During the recent years, the concept of dependence, defined as the level of assistance required by a patient with $\mathrm{AD}$ [17], has been proposed to be an integrative measure to assess the progression of the $\mathrm{AD}$, because it has been shown to decline with the course of the disease [18], and it is associated with cognitive and functional status, and with BPSD [19-20]. Regarding the association between dependence and caregiver burden, there is yet a low number of studies reporting data on the strength of this relationship [21-22]. One of these studies was based on a sample of patients with mild cognitive impairment and patients with mild $\mathrm{AD}$ and showed that a measure of dependence was a good predictor of caregiver burden measured with the ZBI [21]. The other study, using the Caregiver Reaction Assessment scale [23] to assess caregiver 
burden, reported a significant association which remained independent of other factors suggesting that the dependence may more accurately predict caregiver burden that the functional impairment solely [22].

Despite the large body of literature analyzing the factors associated with caregiver burden, and indicating its extensive amount of negative consequences, few studies have used a path analysis approach to determine simultaneously the direct and indirect associations of the caregiver burden with the primary stressors and the contextual factors. The aims of this study were to identify the factors that contribute to the caregiver burden of patients with $\mathrm{AD}$ according to the conceptual framework of dependence, and to explore the direct and indirect relationships that exist between primary and contextual stressors using a path analysis approach.

\section{METHODS}

\section{Participants}

The sample consisted of patients included in the Study of the Cost of Dependence Associated to Alzheimer's disease (the CoDep-AD Study), a study of cost of illness in Spain. The CoDep-AD Study was an observational and cross-sectional multicenter study, performed in 21 memory clinics located in hospitals around different geographical sites of Spain, and was designed to assess the relationship between the economic cost of AD, and the level of dependence. The CoDep-AD Study used a stratified sampling in order to achieve a minimum of 5 patients per centre of each degree of severity according to Clinical Dementia Rating (CDR) [24] score. Patients were included on the basis of a consecutive sampling recruitment procedure in the 
outpatient consultation offices of the study investigators, and subjects were eligible if they met the Diagnostic and Statistical Manual for Mental Disorder (Fourth Edition, Text Revision) [25] criteria for primary degenerative dementia of the Alzheimer type, and the National Institute of Neurological Disorders and Stroke-Alzheimer's Disease and Related Disorder Association criteria for probable AD [26]. Inclusion criteria required patients to have a reliable caregiver. Exclusion criteria were the presence of disability due to other causes than AD (i.e. osteoarthritis), a clinical status that may have a fatal outcome in the short term, and the participation in a clinical trial. In this study, due to the fact that have been reported differences in caregiver burden predictors depending on the type of caregiver [27-28], we only included participants which had a caregiver that was a first-degree family caregiver (spousal or adult child caregivers), and we excluded paid caregivers o other family member caregivers.

\section{Study procedure}

Prior to the recruitment of the participants, all interviewers were trained on the objectives of the study, on the questionnaires and scales administration procedures, and on scoring criteria. Before the raters were allowed to administer the instruments included in the study protocol they needed to complete an online form that included a mock interview with all the questionnaires and scales. Only certified raters participated in the data collection. The study procedure consisted of two visits with the memory clinic staff: one visit with the neurologist, psychiatrist or geriatrist, which collected the demographic and clinical information, and one visit with the neuropsychologist, who administered the questionnaires and scales to the patient and the caregiver in order to assess cognitive function, functional capacities, behavioral and psychological symptoms, dependence, and caregiver burden. The informed consent was obtained from 
all of the participants (patient and/or caregiver) and the study protocol was approved by all the Institutional Review Boards of the participant centers. Data were collected from December 9, 2010, to July 6, 2012.

\section{Measures}

Sociodemographic variables of the caregivers (age, gender, place of residence, work status) and the number of other support caregivers were registered during an interview. The caregiver burden was assessed with the ZBI, which is a 22-items measure of perceived caregiver burden. Each item is scored on a 5-point Likert scale, and total scores range between 0 and 88 points, with higher scores indicating greater burden [13]. The study included a set of standardized and validated instruments. The dependence level was assessed with the Dependence Scale (DS) [29], which is a brief instrument composed of 13 items with a hierarchical structure, with items increasing in

the level of the assistance required by the patients. A global dependence score is derived by summing the scores of all items, ranging from 0 to 15, with higher scores indicating a greater degree of dependence. The cognitive function was assessed with the MiniMental State Examination (MMSE) [30], which provides a brief evaluation of orientation, registration, attention, recall, language, and constructional praxis. Scores range from 0 to 30 points. The ability to perform the ADL was assessed using the Disability Assessment for Dementia (DAD) [31], which is a scale that offers a broad assessment of ADL: basic, instrumental and leisure. It comprises forty items and scores range between 0 and 80 points, which are later transformed into percentages. The behavioral and psychological symptoms were assessed with the Neuropsychiatric Inventory (NPI) [32], which is an instrument designed to measure 12 behavioral and psychological symptoms commonly found in persons with AD. The score for each 
disorder is calculated by multiplying the frequency (1-4) by the severity (1-3), and the sum of all of them provides an overall score (range 0-144). The NPI distress scale is an adjunct scale to the NPI for assessing the impact of the neuropsychiatric symptoms on caregiver distress. This 12-items scale provides a reliable and valid measure of subjective caregiver distress in relation to neuropsychiatric symptoms measured by the NPI [33]. he medical comorbidity was assessed using the Cumulative Illness Rating Scale (CIRS) [34], which consists of 14 items to quantify the burden of chronic diseases taking into account their severity. Score ranges between 0 and 56 points, although a very high score is not biologically plausible, because it would represent the concurrent failure of multiple systems that would not be compatible with life. The dementia severity was assessed using the CDR, which is a semi-structured interview designed to assess the clinical severity of dementia according the level of execution in 6 dimensions (memory, orientation, judgment and problem solving, social leisure and personal care) by an algorithm. The score ranges from 0 (no dementia) to 3 (severe dementia).

\section{Statistical analysis}

We described the clinical and demographic characteristics of the patients and their caregivers by means of absolute and relative frequencies for qualitative variables and by means of central tendency and dispersion measures for quantitative variables. Bivariate analyses of the demographic and clinical characteristics of the patients and caregivers according to the severity of the disease (by using the CDR score as severity classification criteria) were performed using the Kruskall-Wallis test and the $\chi^{2}$ test. Bivariate analyses of the relationship of the caregiver burden and the demographic and clinical characteristics of the patients and caregivers were performed using the Spearman correlation coefficient and the Mann-Whitney test. 
In order to evaluate the fit of the variables related to burden according to the conceptual framework of dependence we used a structural equation modeling approach, and several fit indices were calculated. First, the $\chi^{2}$ test was used, which indicates, when non-significant, that the model and the data are consistent [35]. Second, the Comparative Fit Index (CFI) [36], the Tucker-Lewis Index (TLI) [37], the Root Mean Square Error of Approximation (RMSEA) [38], and the Standardized Root Mean Square Residual (SRMR) [38]. The acceptable model fitting values for these measures were defined as follows: CFI $\geq 0.95$, TLI $\geq 0.95$, RMSEA $<0.05$, SRMR $<0.05$. Third, we calculated the Akaike Information Criterion (AIC) [39], and the Bayesian Information Criterion (BIC) [40]. The AIC and BIC are descriptive indices of the overall goodness of fit. These criteria are based on the distance between a particular model and the model "true" and are the sum of a measure of fit (based on likelihood) and a penalty by the number of model parameters (parsimony). The AIC and BIC indices are not expressed as values between 0 and 1 , and do not have an associated $p$ value. Typically, smaller AIC and BIC values are indicative of a better adjustment when comparing models.

All of the statistical contrasts were bilateral and the confidence intervals (CI) were calculated using a 95\% reliability level. Data processing and analysis was performed using the SPSS statistical program version 19.0 and the Mplus program version 5.0 for Windows. 


\section{RESULTS}

The original sample consisted of 343 patients, the mean age was 78.9 years (SD=7.4) and 67.0\% were women. There were 37 patients with paid caregivers or other non-first degree family members that were excluded for further analyses. There were no differences in the clinical characteristics of the patients according to the type of caregiver, excepting that paid caregivers were younger than first-degree family caregivers (54.1 years [SD=14.0] vs. 61.2 years $[S D=13.9]$; $\mathrm{p}=0.004)$. The final study sample consisted of 306 patients, the mean age was 78.5 years ( $\mathrm{SD}=7.8$ ), and $66.2 \%$ were women. The mean MMSE score was 14.3 points ( $\mathrm{SD}=6.3$ ), the $\mathrm{DAD}$ was 44.2 points ( $\mathrm{SD}=27.7)$, the NPI was 18.3 points ( $\mathrm{SD}=16.4)$, the CIRS was 5.5 points $(\mathrm{SD}=3.9)$, and the $\mathrm{DS}$ was 8.0 points $(\mathrm{SD}=3.2)$. The mean age of caregivers was 61.2 years ( $\mathrm{SD}=13.9), 62.7 \%$ were female, $73.5 \%$ lived with the patient, $32.2 \%$ were employed, and 59.7\% had other support caregivers. The mean ZBI score was 30.7 points $(\mathrm{SD}=16.0)$, and the NPI distress scale was 9.7 points $(\mathrm{SD}=8.9)$. The sociodemographic and clinical characteristics of the study participants according to the severity of AD are shown in Table 1.

The bivariate analyses showed statistical significant differences in the ZBI score depending on the contextual factors: caregiver gender (male/female: 26.3 points $[S D=15.5]$ vs. 33.3 points [SD=15.5]; $\mathrm{p}<0.001$ ), caregiver's place of residence (live with patient/do not live with patient: 32.3 points [SD=16.6] vs. 26.3 points [SD=13.3]; $\mathrm{p}=0.004$ ), and the caregiver's work status (work/no work: 27.5 points [SD=13.9 ] vs. 32.2 points $[S D=16.7] ; p=0.016)$. There were no statistical significant differences in the ZBI score depending on the number of caregivers, nor depending the caregiver's relationship with the patient (partner or children). The stratified analysis according to 
the patient's severity did not show statistical significant differences of the ZBI score regarding the contextual factors (data not shown). For the overall sample, the ZBI score was significantly correlated with the primary stressors: DAD score (Spearman’s rho=0.332; $\mathrm{p}<0.001$ ), NPI score (Spearman’s rho=0.433; $\mathrm{p}<0.001$ ), DS score (Spearman’s rho=0.352; $\mathrm{p}<0.001$ ), and NPI distress scale score (Spearman's rho=0.429; $\mathrm{p}<0.001$ ). In the table 2 are shown the Spearman correlation coefficients between patients and caregivers' characteristics and the ZBI score stratified by dementia severity.

We developed a path model in which caregiver burden was a function of the own caregiver's sociodemographic characteristics (contextual stressors), as well of the dependence level and its clinical determinants, of the disease severity, and of the specific distress related to the BPSD (primary stressors) (figure 1). This model explained $29.9 \%$ of the variance in the ZBI score, and the fit indices values for this model indicated that it fit the data well (table 3). According to this model, the NPI distress scale score, the level of dependence, and the sociodemographic caregiver characteristics were the main direct predictors of caregiver burden. The NPI, and the DAD had important indirect effects via the NPI distress scale, and the DS scale respectively (table 3). Due to the absence of indirect effects of the MMSE score on the ZBI via the CDR or the DS, we developed a second model with a direct relation between these variables (figure 2). This second model explained 32.0\% of the variance in the ZBI, all the fit indices values for this model were better than those of the previous model, and indicated that the model fitted the data well (CFI and TLI values higher than 0.95), and that the model and the data were consistent ( $\chi^{2}$ test of absolute fit was not significant, and SRMR and RMSEA values were lower than 0.05 ). This model provided a reasonable explanation of the direct and indirect relationships between the clinical 
variables of the patient and the caregiver sociodemographic characteristics with the caregiver burden.

\section{DISCUSSION}

The objective of this study was to identify the factors that contribute to the caregiver burden of patients with $\mathrm{AD}$ according to the conceptual framework of dependence. The models that we have examined had an acceptable fit to the data, suggesting that the use of the path analysis approach could be an appropriate strategy to assess the relationships of the stressors with the caregiver burden as proposed by the specific model of stress for dementia [15-16]. Our study identified that the major association with the caregiver burden was related to the primary stressors by direct effects (dependence, cognitive function, disease severity, and distress due to the BPSD), and by indirect effects (functional disability via dependence and disease severity, and BPSD via distress). All the contextual stressors analyzed, excepting the presence of multiple caregivers, were associated with the caregiver burden, but the magnitude of the association was lower than this exhibited by the primary stressors.

It is important to note that this was a naturalistic multicenter study with broad inclusion criteria and specialist clinician diagnoses, which recruited patients within the complete range of the disease severity, from mild to severe cases. An advantage of the present study, taking into account that the caregiver burden changes longitudinally due to the disease progression, is that the design included a stratified sampling in order to achieve a sample of patients within all the disease severity degrees. In this sense, the bivariate analysis demonstrated that the relationship between the caregiver burden and 
the primary stressors was different depending on the disease severity. For example, regarding the relationships of the MMSE, the DAD, the CIRS, and the DS with the ZBI, it is interesting to highlight that the association was variable depending on the disease severity. By contrast, the degree of association of the ZBI score with the contextual factors was not different between the disease severity ranges.

The path analysis allowed us to analyze simultaneously the direct and indirect relationships of the primary stressors and the contextual factors with the caregiver burden. The specific model of stress for dementia caregiver burden proposes to divide factors by its nature (contextual, primary, secondary, and moderator factors). The primary stressors include the cognitive impairment, the functional disability, and the BPSD, and the current literature has used these variables as independent variables and has analyzed its effects on the caregiver burden. The most consistent result is the negative effect of BPSD on the caregiver burden [refs], while regarding the relationship of the cognitive impairment and the functional disability on the caregiver burden is controversial, because some studies have found an association but others failed to identify these variables as significant predictors of the caregiver burden [refs]. In this sense, the use of dependence as a measure of disease progression represents a change of paradigm because, according to the conceptual framework, the dependence level is a result of the cognitive impairment, the functional disability and the BPSD. Thus, the path analysis that we adjusted included as primary stressors the level of dependence and the disease severity, while the cognitive impairment, the functional disability, and the BPSD were the predictors of these primary stressors. This approach permitted to decompose the effects of several independent variables in direct and indirect effects. In concordance with previous studies, the neuropsychiatric symptoms and the related 
distress were the main variables related to the caregiver burden [refs] independently of the rest of stressors. The frequency and severity of the BPSD had an indirect relationship on the caregiver burden via the distress that produce, but also, the distress itself had an important direct relationship. Regarding the level of dependence, our results indicate that it was, after the distress caused by BPSD, the second primary stressor directly related with the caregiver burden irrespective of the disease severity and the contextual factors. The functional disability contributed indirectly to the caregiver burden mainly due to its effects on the dependence level, and in minor degree due to its relationship with the disease severity. The previous study that analyzed relationship between the DS and ZBI identified differences depending on the severity of the cognitive impairment. When all the patients were included in the analysis (backward stepwise multiple regression model), the DS and the NPI were the predictors of the caregiver burden. However, an analysis stratified according the degree of the cognitive impairment, indicated that the DS score and the MMSE score were good predictors of the caregiver burden, but only in patients with a MMSE over 20 points. Instead, for patients with a MMSE less than 20 points, only the NPI score predicted the caregiver burden, and none of the models accepted the functional impairment as independent variable [21]. Our approach, by using the path analysis strategy, overcomes this type of problems, because the standardized path coefficients represent partial regression coefficients that measure the effect of one variable on other, controlling for prior variables.

As stated previously, the association between the cognitive impairment and the caregiver burden has been controversial [refs]. In our study the relationship between the cognitive impairment and the caregiver burden was moderate and positive in the 
bivariate analysis, indicating that better cognitive function increased the caregiver burden, but only for patients with mild severity. The inclusion of a direct relationship in the path analysis between the MMSE score and the caregiver burden resulted in a better model adjustment, incorporating the CDR score as a caregiver burden predictor, and increasing the standardized coefficient of the DS. This result seems paradoxical and merits a more detailed interpretation. The direct effect of the cognitive impairment on the caregiver burden was positive; however the indirect effects via disease severity or via dependence, although not reach the statistical significance, were negative indicating that worse cognitive function increased the caregiver burden. The positive direct relationship between better cognitive function and increased burden, controlled for the rest of stressors in the path analysis, may be interpreted due to the fact that patients have enough cognitive abilities to be more demanding or reiterative, and consequently produces a burdensome effect on the caregiver.

Regarding the contextual factors, the study results indicate that female gender, decreased age, to live with the patient, and do not have a job, were risk factors for an increased caregiver burden independently of the primary stressors. These results are in concordance with the existing literature. Female caregivers reported higher burden than men, and this difference has been attributed to several factors such as the use of more emotion-focused coping strategies [ref], the reduction in social interactions [refs], or the reception of less informal support than men [ref]. The higher burden in caregivers that live with the patient has been previously reported [refs], and has been related to the loss of independence, and due to the increase on the time of the caregiver to attend the patient's necessities. This interpretation is related with our result that indicates that 
caregivers with a job had lower burden, because they spend less time taking in caregiving activities.

Several limitations of this study should be acknowledged. The first limitation is the absence of moderators such as the caregiver's personality traits or coping skills in the path analysis model. According to the theoretical model of caregiver burden, is plausible to expect that the relationships detected in the current study could be modified mainly due to the effect of moderator factors. Second, although the path analysis allowed us to analyze direct and indirect effects simultaneously with multiple independent and dependent variables, the direction of causality between variables was based on the hypothetical relationships expressed within the theoretically-based model. In this sense, it is important to emphasize that the path analysis was based on data from a cross-sectional design and cannot test causal directionality on relationships. Third, we did not assess the presence of secondary stressors, nor the subjective appraisal of the caregivers, limiting the capacity to validate the theoretically-based model of caregiver burden. Fourth, we have used the caregiver burden as a unitary construct measured by the ZBI. However, there is evidence that the caregiver burden may be composed of several dimensions, and the use of a global measure may mask the true relationships between the independent variables and the existing dimensions. The present paper has also a number of strengths, such as using a large sample, which guarantees the confidence in the goodness of fit tests because it doubles the minimum requirement of 10 to 20 individuals for each variable in the model, and ensures the robustness of the statistical analyses in terms of statistical power. Second, the investigators training procedures guarantee an appropriate internal validity of the study results. Third, the 
models were fitted according to a theoretically-based approach, not by using algorithms to adjust the model to the data, conferring to our results a strong external validity.

Future studies should take into account these issues in order to provide a complete picture of the multiple relationships between the independent variables related to the caregiver burden of patients with AD. A longitudinal design with a statistical approach using latent grow curves or mixture modeling may help to quantify the theoretically based model of caregiver burden in dementia, that states that the primary stressors lead to the secondary stressors, and then to the negative appraisal of the situation by the caregiver (exacerbated or mitigated depending of the moderator factors), and finally produces the caregiver burden as a negative outcome. Nevertheless, the results obtained in this study report new evidence about the relationships of the caregiver burden and the level of dependence of the patients, demonstrating that the dependence level is the second most important predictor of the caregiver burden. 
Funding: This study and data collection was sponsored by Janssen Alzheimer Immunotherapy Research \& Development, LLC, and Pfizer Inc. The funder participated in the design of the study. The funder had no part in the analysis, and interpretation of the data; the writing of the report; and the decision to submit the article for publication. Josep Garre-Olmo and Secundino López-Pousa are employees of Institut d’Assistència Sanitària, who were paid contractors to Pfizer Inc and Janssen Alzheimer Immunotherapy Research \& Development, LLC in the development of this manuscript and the study design and data analysis.

Conflict of interest statement: The authors declare that they have no competing interests. None of the authors have interests in any company or institution that might benefit from the publication of this manuscript.

Acknowledgements: We are grateful to all the study participants who have generously given their time and collaborated in the study. We also thank S Monserrat-Vila, for the administrative support, acquisition of data and data handling. 


\section{REFERENCES}

1. Azheimer's Association. 2013 Alzheimer's disease facts and figures. Alzheimers Dement. 2013;9:208-45.

2. Garre-Olmo J, López-Pousa S, Vilalta-Franch J, de Gracia Blanco M, Vilarrasa AB. Grouping and trajectories of neuropsychiatric symptoms in patients with Alzheimer's disease. Part II: two-year patient trajectories. J Alzheimers Dis. 2010;22:1169-1180.

3. Brodaty H, Donkin M. Family caregivers of people with dementia. Dialogues Clin Neurosci. 2009;11:217-228.

4. Etters L, Goodall D, Harrison BE. Caregiver burden among dementia patient caregivers: a review of the literature. J Am Acad Nurse Pract. 2008;20:423-8.

5. Schubert CC, Boustani M, Callahan CM, Perkins AJ, Hui S, Hendrie HC. Acute care utilization by dementia caregivers within urban primary care practices. J Gen Intern Med. 2008;23:1736-1740.

6. Richardson TJ, Lee SJ, Berg-Weger M, Grossberg GT. Caregiver health: health of caregivers of Alzheimer's and other dementia patients. Curr Psychiatry Rep. 2013 Jul;15(7):367

7. García-Alberca JM, Lara JP, Berthier ML. Anxiety and depression in caregivers are associated with patient and caregiver characteristics in Alzheimer's disease. Int J Psychiatry Med. 2011;41:57-69.

8. Sleath B, Thorpe J, Landerman LR, Doyle M, Clipp E. African-American and white caregivers of older adults with dementia: differences in depressive symptomatology and psychotropic drug use. J Am Geriatr Soc 2005;53:397-404. 
9. McCurry SM, Gibbons LE, Logsdon RG, Vitiello MV, Teri L. Insomnia in caregivers of persons with dementia: who is at risk and what can be done about it? Sleep Med Clin 2009;4:519-526.

10. Stevens AB, Coon D, Wisniewski S, Vance D, Arguelles S, Belle S, Mendelsohn A, Ory M, Haley W. Measurement of leisure time satisfaction in family caregivers. Aging Ment Health. 2004;8:450-9.

11. Robison J, Fortinsky R, Kleppinger A, Shugrue N, Porter M. A broader view of family caregiving: effects of caregiving and caregiver conditions on depressive symptoms, health, work, and social isolation. J Gerontol B Psychol Sci Soc Sci. 2009;64:788-798.

12. Vitalino PP. A model of burden in caregivers of DAT patients. In: E Light, BD Lebowitz, eds. Alzheimer 's disease Treatment and Family Stress. New York: Hemisphere Publishing Corporation; 1990:267-291.

13. Zarit SH, Todd PA, Zarit J M. Subjective burden of husbands and wives as caregivers: a longitudinal study. Gerontologist 1986;26:260-266.

14. Bachner YG, O'Rourke N. Reliability generalization of responses by care providers to the Zarit Burden Interview. Aging Ment Health. 2007;11:678-685.

15. Burden theoretical model 1

16. Burden theoretical model 2

17. McLaughlin T, Feldman H, Fillit H, Sano M, Schmitt F, Aisen P, Leibman C, Mucha L, Ryan JM, Sullivan SD, Spackman DE, Neumann PJ, Cohen J, Stern Y. Dependence as a unifying construct in defining Alzheimer's disease severity. Alzheimers Dement. 2010;6:482-93. 
18. Brickman AM, Riba A, Bell K, Marder K, Albert M, Brandt J, Stern Y. Longitudinal assessment of patient dependence in Alzheimer disease. Arch Neurol. 2002;59:1304-1308.

19. McLaughlin T, Buxton M, Mittendorf T, Redekop W, Mucha L, Darba J, Jonsson L, Lacey L, Leibman C. Assessment of potential measures in models of progression in Alzheimer disease. Neurology. 2010;75:1256-62.

20. Spackman DE, Kadiyala S, Neumann PJ, Veenstra DL, Sullivan SD. The validity of dependence as a health outcome measure in Alzheimer's disease. Am J Alzheimers Dis Other Demen. 2013;28:245-52.

21. Gallagher D, AN Mhaolain A, Crosby L, Ryan D, Lacey L, Coen RF, Walsh C, Coakley D, Walsh JB, Cunningham C, Lawlor BA. Dependence and caregiver burden in Alzheimer's disease and mild cognitive impairment. Am J Alzheimers Dis Other Demen. 2011;26:110-114.

22. Lacey L, McLaughlin TP, Mucha L, Grundman M, Black R. Relationship between patient dependence on others and caregiver burden in Alzheimer's Disease. Alzeimers Dement 2009;5(Suppl):P229.

23. Given CW, Given B, Stommel M, Collins C, King S, Franklin S. The caregiver reaction assessment (CRA) for caregivers to persons with chronic physical and mental impairments. Res Nurs Health. 1992;15:271-283.

24. Morris JC. The Clinical Dementia Rating (CDR): current version and scoring rules. Neurology 1993;43:2412-2414.

25. American Psychological Association. Diagnostic and Statistical Manual of Mental Disorder Revised 4 ed, American Psychiatric Press: Washington, DC, 1994. 
26. McKhann G, Drachman D, Folstein M, Katzman R, Price D, Stadlan EM. Clinical diagnosis of Alzheimer's disease: report of the NINCDS-ADRDA Work Group under the auspices of Department of Health and Human Services Task Force on Alzheimer's Disease. Neurology. 1984;34:939-944.

27. Rosa E, Ambrogio M, Binetti G, Zanetti O. 'Immigrant paid caregivers' and primary caregivers' burden. Int J Geriatr Psychiatry. 2004;19(:1103-1104.

28. Lin WC, Tsai CF, Wang SJ, Hwang JP, Fuh JL. Comparison of the burdens of family caregivers and foreign paid caregivers of the individuals with dementia. Int Psychogeriatr. 2012;24:1953-1961.

29. Stern Y, Albert SM, Sano M, Richards M, Miller L, Folstein M, Albert M, Bylsma FW, Lafleche G. Assessing patient dependence in Alzheimer's disease. J Gerontol. 1994;49:M216-22.

30. Folstein MF, Folstein SE, McHugh PR. "Mini Mental State". A practical method for grading the cognitive state of patients for the clinician. J Psychiatry Research 1975;12: 189-198.

31. Gélinas I, Gauthier L, McIntyre M, Gauthier S. Development of a functional measure for persons with Alzheimer’s disease: the Disability Assessment for Dementia. Am J Occup Ther 1999;53:471-481.

32. Cummings JL, Mega M, Gray K, Rosenberg-Thompson S, Carusi DA, Gornbein J. The neuropsychiatric inventory. Comprehensive assessment of psychopathology in dementia. Neurology 1994;44:2308-14.

33. Kaufer DI, Cummings JL, Christine D, Bray T, Castellon S, Masterman D, MacMillan A, Ketchel P, DeKosky ST. Assessing the impact of neuropsychiatric 
symptoms in Alzheimer's disease: the Neuropsychiatric Inventory Caregiver Distress Scale. J Am Geriatr Soc. 1998;46:210-5.

34. Conwell Y, Forbes NT, Cox C, Caine ED. Validation of a measure of physical illness burden at autopsy: the Cumulative Illness Rating Scale. J Am Geriatr Soc. $1993 ; 41: 38-41$

35. Kaplan D. Path Analysis: Modeling Systems of Structural Equations Among Observed Variables. In Structural Equation Modeling: Foundations and Extensions. (2nd. Ed.): Sage Publications. Thousand Oaks, 2009.

36. Bentler PM (1990) Comparative fit indexes in structural models. Psychol Bull 107, 238-246.

37. Tucker LR, Lewis C (1973) A reliability coefficient for maximum likelihood factor analysis. Psychometrika 38, 1-10.

38. Browne MW, Cudeck R (1993) Alternative ways of assessing model fit. In Testing structural equation models, Bollen KA y Long JS, eds. Sage, Thousand Oaks, pp. 136-162.

39. Akaike H (1987) Factor analysis and AIC. Psychometrika 52, 317-322.

40. Schwartz G (1978) Estimating the dimension of a model. Ann Statistics 6, 461464. 
Table 1. Demographic and clinical characteristics of patients and caregivers according to dementia severity

\begin{tabular}{lcccc}
\hline CDR score & $\mathbf{1}$ & $\mathbf{2}$ & $\mathbf{3}$ & \\
& $(\mathrm{n}=102)$ & $(\mathrm{n}=110)$ & $(\mathrm{n}=94)$ & $\mathbf{p}$ \\
\hline Patients characteristics & & & & \\
Age (years), mean (SD) & $76.4(7.3)$ & $79.5(6.5)$ & $79.6(8.3)$ & 0.002 \\
Gender (female), n (\%)* & $68(67.7)$ & $71(64.5)$ & $63(68.5)$ & 0.839 \\
MMSE score, mean (SD) & $19.1(4.3)$ & $15.1(4.1)$ & $7.9(5.0)$ & $<0.001$ \\
DAD score, mean (SD) & $71.1(17.1)$ & $43.0(18.2)$ & $16.4(15.5)$ & $<0.001$ \\
NPI score, mean (SD) & $12.8(12.2)$ & $18.0(15.2)$ & $25.0(19.6)$ & $<0.001$ \\
CIRS score, mean (SD) & $5.4(3.3)$ & $5.4(3.7)$ & $5.7(4.4)$ & 0.838 \\
DS score, mean (SD) & $5.0(2.4)$ & $8.2(2.0)$ & $11.0(2.1)$ & $<0.001$
\end{tabular}

\section{Caregivers characteristics}

\begin{tabular}{|c|c|c|c|c|}
\hline Age, mean (SD) & $61.6(14.0)$ & $60.7(14.5)$ & $61.3(13.2)$ & 0.903 \\
\hline Gender (female), n (\%) & 58 (56.9) & $72(65.5)$ & $60(65.2)$ & 0.353 \\
\hline Living together, n (\%) & $69(67.7)$ & $86(78.2)$ & $69(75.0)$ & 0.207 \\
\hline Employed*, n (\%) & $36(35.5)$ & $31(28.2)$ & $30(33.0)$ & 0.500 \\
\hline Multiple caregivers*, n (\%) & $50(50.0)$ & $66(60.6)$ & $63(68.5)$ & 0.032 \\
\hline NPI distress scale, mean (SD) & $7.5(7.4)$ & $10.3(8.8)$ & $11.4(10.2)$ & 0.007 \\
\hline ZBI score, mean (SD) & $24.2(14.3)$ & $33.1(15.4)$ & $35.0(16.5)$ & $<0.001$ \\
\hline
\end{tabular}


Table 2. Spearman correlation coefficients between patient and caregiver characteristics and caregiver burden stratified by dementia severity

\begin{tabular}{lccc}
\hline CDR score & $\mathbf{1}$ & $\mathbf{2}$ & $\mathbf{3}$ \\
& $(\mathrm{n}=102)$ & $(\mathrm{n}=110)$ & $(\mathrm{n}=94)$ \\
\hline Patients characteristics & & & \\
Age (years) & -0.012 & 0.132 & 0.030 \\
MMSE score & $0.260^{\dagger}$ & 0.056 & 0.067 \\
DAD score & -0.184 & $-0.278^{\dagger}$ & -0.109 \\
NPI score & $0.465^{\ddagger}$ & $0.497^{\ddagger}$ & $0.210^{\dagger}$ \\
CIRS score & $0.237^{\dagger}$ & 0.148 & -0.107 \\
DS score & $0.293^{\dagger}$ & $0.227^{\dagger}$ & 0.175
\end{tabular}

\section{Caregivers characteristics}

$\begin{array}{llll}\text { Age, mean (SD) } & -0.137 & -0.068 & 0.159 \\ \text { NPI distress scale, mean (SD) } & 0.431^{\ddagger} & 0.528^{\ddagger} & 0.209^{\dagger}\end{array}$

\footnotetext{
* Data not available in 2 cases; CDR: Clinical Dementia Rating; MMSE: Mini-Mental State Examination;

DAD: Disability Assessment for Dementia; NPI: Neuropsychiatric Inventory; CIRS: Cumulative Illness

Rating Scale; ZBI: Zarit Burden Interview European Quality of Life-5 Dimensions; DS: Dependence Scale;

${ }^{\dagger}: p<0.05 ;{ }^{\ddagger}: p<0.001$
} 
Table 4. Standardized indirect relationships with caregiver burden

\begin{tabular}{|c|c|c|}
\hline Relation with ZBI & Indirect model 1 (SE) & Indirect model 2 (SE) \\
\hline CDR* & $0.014(0.010)$ & $0.017(0.012)$ \\
\hline CIRS* & $0.012(0.007)$ & $0.015(0.008)$ \\
\hline Patient age* & $0.016(0.008)$ & $0.020^{\dagger}(0.009)$ \\
\hline MMSE via CDR & $-0.016(0.024)$ & $-0.053(0.027)$ \\
\hline MMSE via DS & $-0.009(0.008)$ & $-0.011(0.009)$ \\
\hline MMSE via CDR \& DS & $-0.004(0.003)$ & $-0.006(0.004)$ \\
\hline DAD via CDR & $-0.028(0.040)$ & $-0.091^{\dagger}(0.046)$ \\
\hline DAD via DS & $-0.154^{\dagger *}(0.060)$ & $-0.190^{\dagger}(0.061)$ \\
\hline DAD via CDR \& DS & $-0.008(0.006)$ & $-0.010(0.007)$ \\
\hline NPI via CDR & $0.003(0.004)$ & $0.009(0.007)$ \\
\hline NPI via DS & $0.013(0.007)$ & $0.016(0.008)$ \\
\hline NPI via CDR \& DS & $0.001(0.001)$ & $0.001(0.001)$ \\
\hline NPI via distress & $0.269^{\dagger}(0.042)$ & $0.258^{\dagger}(0.042)$ \\
\hline
\end{tabular}

SE: Standard error; DS: Dependence Scale; CDR: Clinical Dementia Rating; CIRS: Cumulative Illness Rating Scale; MMSE: Mini-Mental State Examination; DAD: Disability Assessment for Dementia; NPI: 
Neuropsychiatric Inventory; *: Indirect relationship via DS; ${ }^{\dagger}: p<0.05$

\section{Figure Legends}

Figure 1. Model of caregiver burden according to the conceptual framework of dependence (non-significant path coefficients are not shown)

Figure 2. Modified model of burden according to the conceptual framework of dependence (non-significant path coefficients are not shown) 


\section{Figure 1}

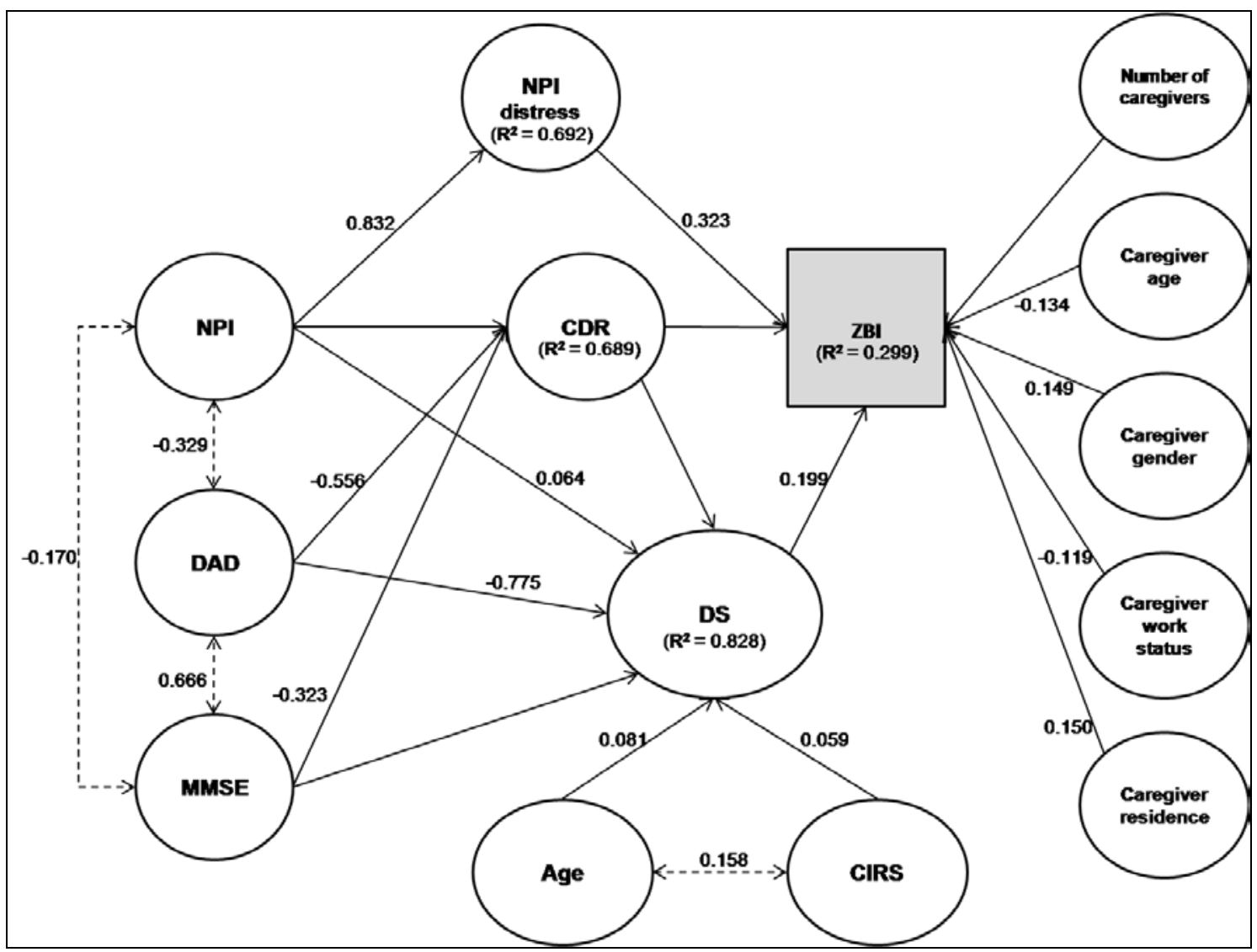

CDR: Clinical Dementia Rating; MMSE: Mini-Mental State Examination; DAD: Disability Assessment for Dementia; NPI: Neuropsychiatric Inventory; CIRS: Cumulative Illness Rating Scale; DS: Dependence Scale; ZBI: Zarit Burden Interview; $\mathrm{R}^{2}$ : Coefficient of determination 


\section{Figure 2}

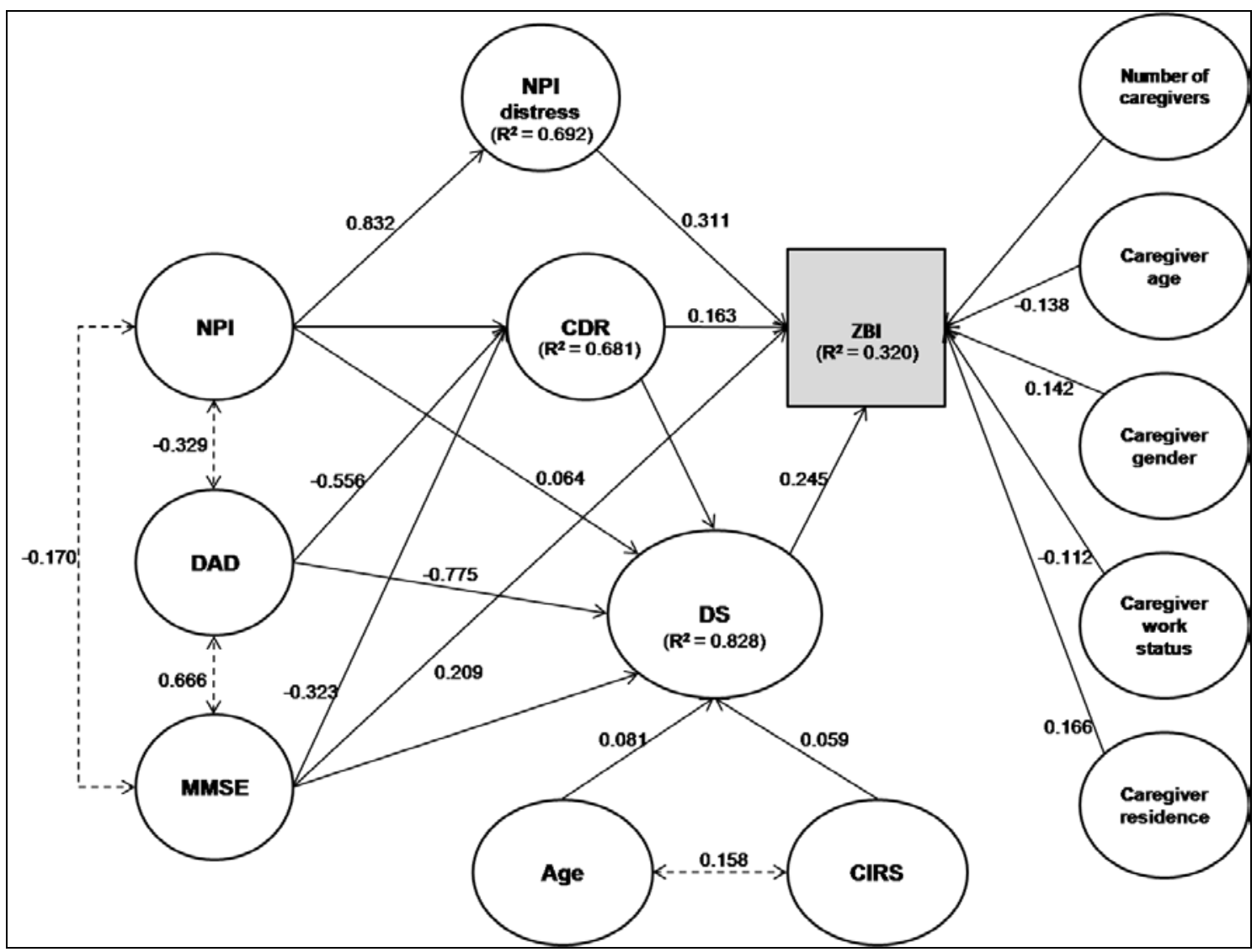

CDR: Clinical Dementia Rating; MMSE: Mini-Mental State Examination; DAD: Disability Assessment for Dementia; NPI: Neuropsychiatric Inventory; CIRS: Cumulative Illness Rating Scale; DS: Dependence Scale; ZBI: Zarit Burden Interview; ${ }^{2}$ : Coefficient of determination 